\title{
Valence-loss EELS Spectroscopy of Refractory Plasmonic Nanomaterials
}

\author{
Andrew A. Herzing ${ }^{1}$, Urcan Guler ${ }^{2}$, Xiuli Zhou ${ }^{3}$ and Theodore B. Norris ${ }^{3}$ \\ 1. Material Measurement Laboratory, National Institute of Standards and Technology, Gaithersburg, MD \\ USA. \\ 2. School of Electrical \& Computer Engineering, Purdue University, West Lafayette, IN USA. \\ 3. Center for Ultrafast Optical Science, University of Michigan, Ann Arbor, MI USA.
}

The interaction of light with nanostructures via plasmonic resonance offers a promising route to controlling the properties and propagation of light at length scales which are far below the diffraction limit. These nanostructures typically consist of noble metals, and can exhibit very strong, well-defined resonances. However, the promise of this technology has yet to be fully realized, which is due in large part to inherent limitations in the chosen materials, which often result in heavy losses in the optical range. Similarly, they can suffer from poor chemical and/or thermal stability, which severely restricts their use in practical applications. More recently, refractory transition metal nitrides have been proposed as a promising class of alternative materials for plasmonic applications. In particular, TiN has been found to exhibit ideal properties for many applications [1-3]. This material exhibits similar optical properties to those of $\mathrm{Au}$, but is much more thermally stable, less expensive, and is compatible with traditional semiconductor fabrication technology. Herein, we report measurements of the local plasmonic response of TiN thin-films and nanocubes via monochromated electron energy-loss spectroscopy (EELS) in the scanning transmission electron microscope (STEM).

$150 \mathrm{~nm}$ thick films of TiN were deposited on an $\mathrm{MgO}$ substrate via DC magnetron sputtering of a titanium target in an argon-nitrogen environment. The base pressure was $8 \times 10^{-8}$ Torr and the substrate temperature during deposition was $800{ }^{\circ} \mathrm{C}$. Cross-sectional specimens of the TiN film were prepared using a focused ion-beam (FIB) lift-out procedure after first protecting the TiN film surface by sputter-coating it with 25 $\mathrm{nm}$ of $\mathrm{Au}-\mathrm{Pd}$. This layer protects the surface during the deposition of a further Pt overlayer in the FIB prior to cross-sectional milling. Samples were also prepared from a solution of TiN nanocubes suspended in deionized water by drop casting onto a $15 \mathrm{~nm}$ thick silicon nitride membrane.

The inset of Figure 1 shows a STEM-HAADF image of the MgO-TiN interface acquired with the crystals oriented along the <001> direction. The overall quality of the TiN film is quite high and is seen to grow epitaxially from the $\mathrm{MgO}$ substrate. Electron energy-loss spectroscopy (EELS) was carried out using a monochromated $150 \mathrm{keV}$ electron beam with a spectral energy-loss resolution of $\approx 0.15 \mathrm{eV}$ as measured by the FWHM of the zero-loss peak. EELS spectra of the valence loss response were acquired from the specimen using a monochromated probe with a current of 20 pA. Spectra were collected along a line beginning in the $\mathrm{MgO}$ substrate and terminating in the protective Pt overlayer. The energy-axis of all spectra were then aligned, and 20 spectra were integrated from the $\mathrm{MgO}$ substrate, $\mathrm{MgO} / \mathrm{TiN}$ interface, and the center of the TiN film (Fig. 1). The spectrum from the MgO substrate exhibits features typical of this material [4] with a bandgap onset indicated by the abrupt increase in inelastic scattering near $7.5 \mathrm{eV}$. At the junction of the $\mathrm{MgO}$ and TiN film, a relatively sharp peak was observed at $(2.05 \pm 0.01) \mathrm{eV}$. Further from the interface, at the center of the TiN film, a weaker peak was observed at $(2.81 \pm 0.3) \mathrm{eV}$. This peak was followed by a nearly linear increase in inelastic scattering intensity that was continuous up until a discrete change near $11 \mathrm{eV}$. Multiple acquisitions from random points in the film showed that the spectral response did not vary with position.

Similar results from the TiN nanocubes are shown in Figure 2. In this case, the spectral response from the interior of the particle nicely matches that collected from the center of the TiN film in Figure 1, and 
represents a combination of the bulk and surface excitation with the former being much stronger than the latter. Subtle differences are observed in the low-loss response collected from the particle corner versus that collected near the cube face. These differences can be understood by comparison to optical simulations, and were found to be due to particle shape and surface chemistry effects.

\section{References:}

[1] U. Guler, et al, Appl. Phys. B 107 (2012) 285.

[2] U. Guler, et al, Nano Letters 13 (2013) 6078.

[3] G. V. Naik, et al, Opt. Mater. Express 2 (2012) 478.

[4] C. C. Ahn and O. L. Krivanek, "EELS Atlas”, Gatan, 1983.

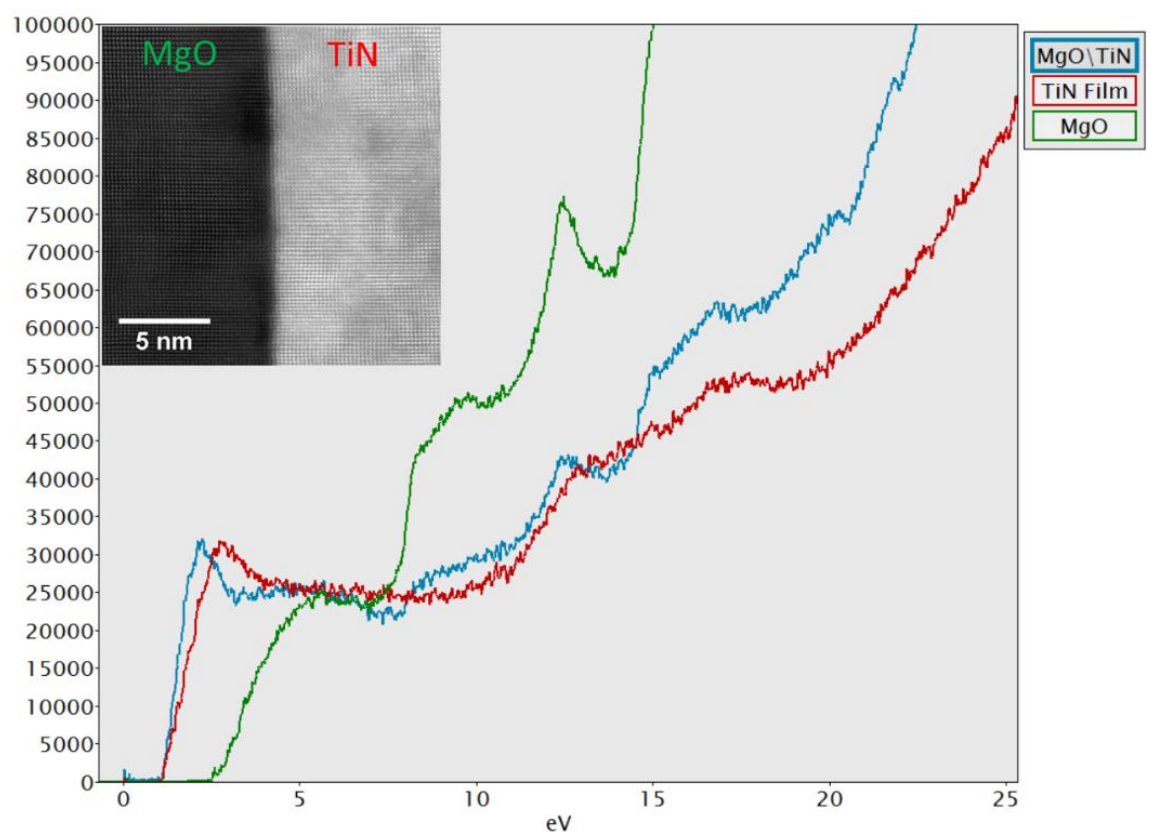

Figure 1. Low-loss STEMEELS analysis of $\mathrm{MgO}$ supported TiN thin film cross-sectional specimen. EELS spectra were collected from various points in the region depicted by the HAADF image (inset).

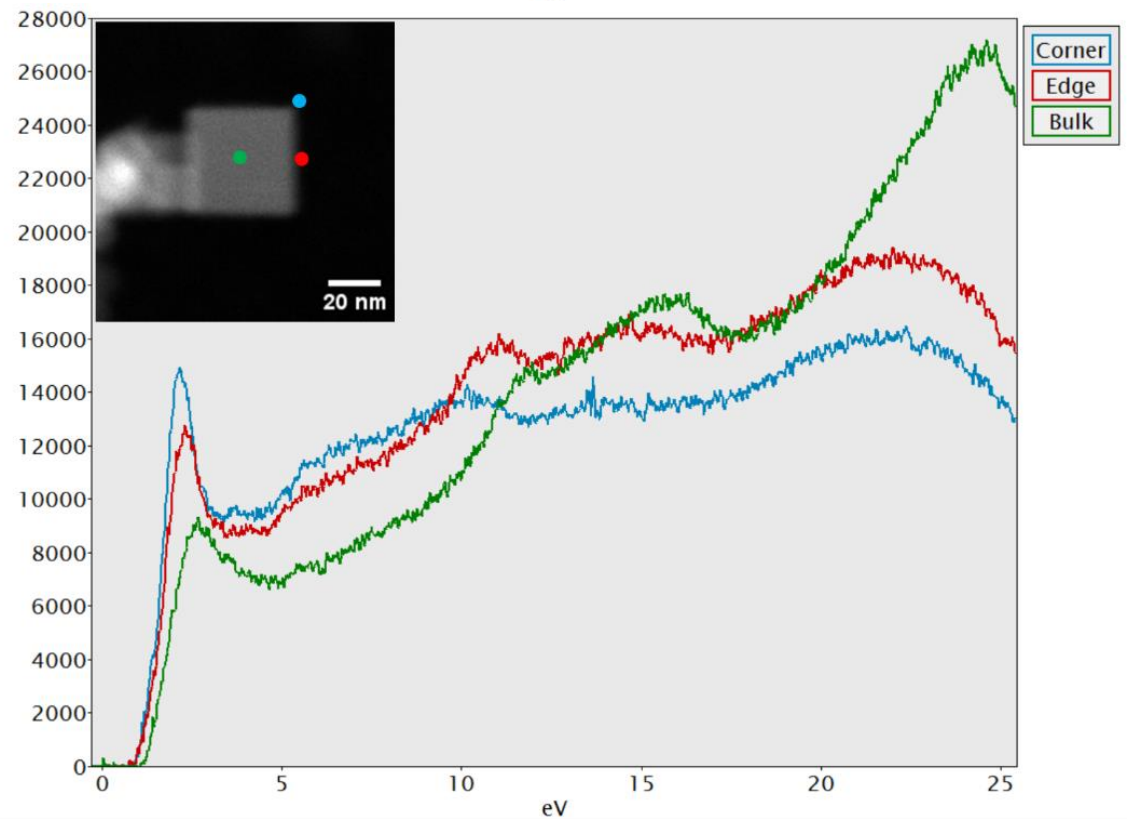

Figure 2. Low-loss STEMEELS analysis of a single TiN nanocube shown in the HAADF image (inset). EELS spectra were extracted from a 2D spectrum image at the points shown by the colored dots. 